\title{
PERFECTNESS IN LOCALES
}

\author{
JAVIER GUTIÉRREZ GARCÍA, TOMASZ KUBIAK, AND JORGE PICADO
}

Dedicated to Professor Bernhard Banaschewski on the occasion of his $90^{\text {th }}$ birthday

\begin{abstract}
Aвstract. This paper makes a comparison between two notions of perfectness for locales which come as direct reformulations of the two equivalent topological definitions of perfectness. These reformulations are no longer equivalent. It will be documented that a locale may appropriately be called perfect if each of its open sublocales is a join of countably many closed sublocales. Certain circumstances are exhibited in which both reformulations coincide. This paper also studies perfectness in mildly normal locales. It is shown that perfect and mildly normal locales coincide with the Oz locales extensively studied in the last decade.
\end{abstract}

\section{INTRODUCTION}

In this paper we look for the extension to the pointfree setting of what in topology is called perfectness. We recall that a topological space is called perfect if each open set is a union of countably many closed sets, i.e. open sets are $F_{\sigma}$. This is equivalent to the statement that each closed set is an intersection of countably many open sets, i.e. closed sets are $G_{\delta}$. The two equivalent formulations of perfectness for spaces have direct reformulations for locales in terms of open sublocales and closed sublocales. The two resulting concepts, which will be called $F_{\sigma}$-perfectness and $G_{\delta}$-perfectness, are no longer equivalent, for $G_{\delta}$-perfectness is generally stronger than $F_{\sigma}$-perfectness.

The first purpose of this paper is to compare those two non-equivalent concepts. One nice feature of $F_{\sigma}$-perfectness is that the locale $O X$ of all open sets of an arbitrary perfect space $X$ is always $F_{\sigma}$-perfect, but may fail to be $G_{\delta}$-perfect (Section 3); another one is that it behaves nicely with respect to closed maps (Section 5). This shows that, with respect to the criterion of conservativeness, $F_{\sigma}$-perfectness behaves much better than $G_{\delta}$-perfectness. Moreover, $F_{\sigma}$-perfectness will be shown to be conservative in a quite large class of spaces (containing all $T_{1}$-spaces). In the class of normal locales, $F_{\sigma}$-perfectness and $G_{\delta}$-perfectness coincide, and are conservative concepts for the class

Date: June 20, 2016.

2010 Mathematics Subject Classification. 06D22, 54D15.

Key words and phrases. Locale, sublocale, $F_{\sigma}$-sublocale, $G_{\delta}$-sublocale, normality, mild normality, perfectness, perfect normality, pm-normality, Oz locale. 
of perfect $T_{0}$-spaces. Due to all those circumstances we eventually drop the prefix $F_{\sigma^{-}}$ and call a locale perfect if each its open sublocale is a join of countably many closed sublocales.

The second purpose of this paper is to study perfectness in mildly normal locales (Section 4). One interesting observation is that perfect and mildly normal locales coincide with the so-called $\mathrm{Oz}$ locales extensively studied in the last decade (cf. [1, 2, 5, 6]).

\section{Preliminaries on locales}

For general background regarding locales and frames we refer to [12] or [15]. Here, we present a brief outline of the facts specifically needed for the paper.

A locale or a frame is a complete lattice $L$ in which

$$
a \wedge \bigvee B=\bigvee\{a \wedge b: b \in B\}
$$

for all $a \in L$ and $B \subseteq L$. The topology of a topological space $X$ is a locale and is denoted by $O(X)$. Being a Heyting algebra, each locale $L$ has the implication operator

$$
a \rightarrow b=\bigvee\{x \in L \mid x \wedge a \leq b\}
$$

satisfying the standard equivalence $c \wedge a \leq b$ iff $c \leq a \rightarrow b$. The pseudocomplement of an $a \in L$ is the element $a^{*}=a \rightarrow 0$. An element $a$ is regular if $a^{* *}=a$ (equivalently, if $a=b^{*}$ for some $\left.b \in L\right)$. Note that the first De Morgan law $(a \vee b)^{*}=a^{*} \wedge b^{*}$ holds in any locale (actually, more generally, $\left.(\bigvee A)^{*}=\bigwedge_{a \in A} a^{*}\right)$.

For any elements $a$ and $b$ in $L, a<b$ ( $a$ is well inside $b$ ) means that $a \wedge x=0$ and $b \vee x=1$ for some $x \in L$ (equivalently, $a^{*} \vee b=1$ ).

A sublocale $S$ of a locale $L$ is a subset $S \subseteq L$ such that:

(S1) for every $A \subseteq S, \wedge A$ is in $S$, and

(S2) for every $s \in S$ and every $x \in L, x \rightarrow s$ is in $S$.

The set $\mathcal{S}(L)$ of all sublocales of $L$ forms a co-frame (i.e., the dual lattice is a frame) under inclusion, in which arbitrary infima coincide with intersections. Regarding suprema, there is the formula

$$
\bigvee_{i \in I} S_{i}=\left\{\bigwedge A: A \subseteq \bigcup_{i \in I} S_{i}\right\}
$$

for every $\left\{S_{i} \in \mathcal{S}(L): i \in I\right\}$.

Since $\mathcal{S}(L)$ is the dual of a complete Heyting algebra, it has co-pseudocomplements, given by the formula

$$
S^{\#}=L \backslash S=\bigcap\{T \in \mathcal{S}(L) \mid S \vee T=L\} .
$$

Note that

$$
\left(\bigcap_{i \in I} S_{i}\right)^{\#}=\bigvee_{i \in I} S_{i}^{\#}, \quad\left\{S_{i}\right\}_{i \in I} \subseteq \mathcal{S}(L) .
$$


For each $a \in L$, the sublocales $\mathfrak{c}(a)=\uparrow a$ and $\mathfrak{D}(a)=\{a \rightarrow b \mid b \in L\}$ are the closed and open sublocales of $L$ induced by $a$, respectively. We summarize here the basic properties of sublocales used throughout the paper:

(P1) For every $a \in L, \mathfrak{c}(a)$ and $\mathfrak{v}(a)$ are complements of each other in $\mathcal{S}(L)$.

(P2) For every $a, b \in L, \mathfrak{c}(b) \subseteq \mathfrak{v}(a)$ if and only if $a \vee b=1$ and $\mathfrak{c}(b) \supseteq \mathfrak{v}(a)$ iff $a \wedge b=0$.

(P3) For every $A \subseteq L, \bigvee_{a \in A} \mathfrak{p}(a)=\mathfrak{p}(\bigvee A)$ and $\bigcap_{a \in A} \mathfrak{c}(a)=\mathfrak{c}(\bigvee A)$

\section{A COMPARISON BETWEEN $F_{\sigma}$-PERFECTNESS AND $G_{\delta}$-PERFECTNESS}

Definition 3.1. A locale $L$ is said to be:

(1) $F_{\sigma}$-perfect whenever any open sublocale of $L$ is an $F_{\sigma}$-sublocale, that is, for each $a \in L$ there exists a countable family $\left(a_{n}\right)_{n \in \mathbb{N}}$ in $L$ such that

$$
\mathfrak{v}(a)=\bigvee_{n \in \mathbb{N}} \mathfrak{c}\left(a_{n}\right)
$$

(2) $G_{\delta}$-perfect if any closed sublocale is a $G_{\delta}$-sublocale, that is, for each $a \in L$ there exists a countable family $\left(a_{n}\right)_{n \in \mathbb{N}}$ in $L$ such that

$$
\mathfrak{c}(a)=\bigcap_{n \in \mathbb{N}} \mathfrak{p}\left(a_{n}\right)
$$

By [15, Proposition V.1.4], each $F_{\sigma}$-perfect locale is subfit, and by [15, Proposition V.1.3.2], each $G_{\delta}$-perfect locale is fit. We recall that a locale is subfit (resp. fit) if every open (resp. closed) sublocale is a join of closed sublocales (resp. meet of open sublocales).

Since $\mathcal{S}(L)$ is no longer a (complete) Boolean algebra, it is not surprising that these two concepts are not equivalent, in general. More specifically, by (\#) and (P1) we have:

Remark 3.2. Each $G_{\delta}$-perfect locale is $F_{\sigma}$-perfect.

However, the converse is far from being true. The following example shows that a $F_{\sigma}$-perfect locale need not even be fit.

Example 3.3. Let $\mathbb{N}$ be endowed with the cofinite topology

$$
O \mathbb{N}=\{\varnothing\} \cup\{U \subseteq \mathbb{N} \mid \mathbb{N} \backslash U \text { finite }\}
$$

For each $U, V \in O \mathbb{N}$ we have

$$
U \rightarrow V= \begin{cases}\mathbb{N}, & \text { if } U=\varnothing ; \\ \operatorname{Int}(\mathbb{N} \backslash U)=\varnothing, & \text { if } U \neq \varnothing=V ; \\ \operatorname{Int}((\mathbb{N} \backslash U) \cup V)=(\mathbb{N} \backslash U) \cup V, & \text { if } U, V \neq \varnothing .\end{cases}
$$

Hence

$$
U \rightarrow V=V \Longleftrightarrow(U=\varnothing \text { and } V=\mathbb{N}) \text { or }(U \neq \varnothing=V) \text { or }(U \neq \varnothing \text { and } \mathbb{N} \backslash U \subseteq V) \text {. }
$$


Consequently, $\mathfrak{p}(\varnothing)=\{\mathbb{N}\}$ and, for each $\varnothing \neq U \in O \mathbb{N}$,

$$
\mathfrak{o}(U)=\{\varnothing\} \cup\{V \in O \mathbb{N} \mid \mathbb{N} \backslash U \subseteq V\}=\{\varnothing\} \cup\{V \in O \mathbb{N} \mid U \cup V=\mathbb{N}\} .
$$

We shall now prove that

$$
\mathfrak{p}(U)=\bigvee_{n \in U} \mathfrak{c}(\mathbb{N} \backslash\{n\}),
$$

for every $U \in O \mathbb{N}$. For $U=\varnothing$ this is trivial since $\mathfrak{p}(\varnothing)=\{\mathbb{N}\}$ is the bottom element of $\mathcal{S}(O \mathbb{N})$. Further, let $\varnothing \neq U \in O \mathbb{N}$ and $n \in U$. Then $\mathfrak{c}(\mathbb{N} \backslash\{n\})=\{\mathbb{N}, \mathbb{N} \backslash\{n\}\} \subseteq \mathfrak{p}(U)$ and $\bigvee_{n \in U} \mathfrak{c}(\mathbb{N} \backslash\{n\}) \subseteq \mathfrak{p}(U)$. Conversely, we first notice that

$$
\varnothing=\operatorname{Int}(\mathbb{N} \backslash U)=\operatorname{Int}\left(\bigcap_{n \in U}(\mathbb{N} \backslash\{n\})\right)=\bigwedge_{n \in U}(\mathbb{N} \backslash\{n\}) \in \bigvee_{n \in U} c(\mathbb{N} \backslash\{n\})
$$

Finally, for each $\varnothing \neq V \in \mathfrak{p}(U)$ we have

$$
V=\operatorname{Int} V=\operatorname{Int}\left(\bigcap_{n \in \mathbb{N} \backslash V}(\mathbb{N} \backslash\{n\})\right)=\bigwedge_{n \in \mathbb{N} \backslash V}(\mathbb{N} \backslash\{n\}) \in \bigvee_{n \in U} c(\mathbb{N} \backslash\{n\}) .
$$

We conclude that $\mathfrak{p}(U) \subseteq \bigvee_{n \in U} \mathfrak{c}(\mathbb{N} \backslash\{n\})$. Hence $\mathfrak{p}(U)=\bigvee_{n \in U} \mathfrak{c}(\mathbb{N} \backslash\{n\})$ is an $F_{\sigma^{-}}$ sublocale which shows that $O \mathbb{N}$ is an $F_{\sigma}$-perfect locale.

On the other hand, the only closed sublocales of $O \mathbb{N}$ which are meets of open sublocales are $\mathfrak{c}(\mathbb{N})$ and $\mathfrak{c}(\varnothing)$ and thus $O \mathbb{N}$ is not fit, hence neither $G_{\delta}$-perfect.

Let us recall that a localic property $L P$ is a conservative extension of a topological property $P$ if, given a topological space $X$, the locale $O(X)$ has property $L P$ if and only if $X$ has property $P$.

Since the space $\left(\mathbb{N}, O \mathbb{N}\right.$ ) is perfect (as any countable $T_{1}$-space does), $G_{\delta}$-perfectness is not a conservative extension of topological perfectness. Unlike $G_{\delta}$-perfectness, the following holds:

Proposition 3.4. If a space $X$ is perfect, then $O X$ is $F_{\sigma}$-perfect.

Proof. Let $U \in O X$. By hypothesis, there exists a countable family $\left(U_{n}\right)_{n \in \mathbb{N}}$ in $O X$ such that $U=\bigcup_{n \in \mathbb{N}}\left(X \backslash U_{n}\right)$. It follows that $U_{n} \cup U=X$ for each $n \in \mathbb{N}$ and thus $\bigvee_{n \in \mathbb{N}} \mathfrak{c}\left(U_{n}\right) \subseteq \mathfrak{p}(U)$ by (P2). On the other hand, let $V \in \mathfrak{p}(U)$ and $V_{n}=U_{n} \cup V \in \mathfrak{c}\left(U_{n}\right)$ for each $n \in \mathbb{N}$, then

$$
\begin{aligned}
V & =U \rightarrow V=\operatorname{Int}((X \backslash U) \cup V)=\operatorname{Int}\left(\left(\bigcap_{n \in \mathbb{N}} U_{n}\right) \cup V\right)=\operatorname{Int}\left(\bigcap_{n \in \mathbb{N}}\left(U_{n} \cup V\right)\right) \\
& =\operatorname{Int}\left(\bigcap_{n \in \mathbb{N}} V_{n}\right)=\bigwedge_{n \in \mathbb{N}} V_{n} \in \bigvee_{n \in \mathbb{N}} \mathfrak{c}\left(U_{n}\right) .
\end{aligned}
$$

Hence $\mathfrak{v}(U) \subseteq \bigvee_{n \in \mathbb{N}} \mathfrak{c}\left(U_{n}\right)$ and we conclude that $O X$ is an $F_{\sigma}$-perfect locale.

The converse implication is not true in general, as shown by the following example: 
Example 3.5. Let $X$ be a $T_{1}$ topological space, $\infty \notin X, Y=X \cup\{\infty\}$ and

$$
O Y=\{\varnothing\} \cup\{U \cup\{\infty\} \mid \varnothing \neq U \in O X\} .
$$

$O Y$ is a topology in $Y$ if and only if $\varnothing$ is meet-irreducible in $O X$. In this case $O Y$ and $O X$ are clearly isomorphic locales and consequently $O Y$ is an $F_{\sigma}$-perfect locale if and only if so is $O X$. Moreover, the space $Y$ is perfect if and only if $X$ is indiscrete. Consequently, if $X$ is a non-indiscrete and perfect topological space such that $\varnothing$ is meet-irreducible in $O X$ (e.g. $\mathbb{N}$ endowed with the cofinite topology), it follows from Proposition 3.4 that $O X$ is an $F_{\sigma}$-perfect locale and hence so is $O Y$. However, $Y$ fails to be perfect.

This construction of space $Y$ is related with space $\Sigma$ in [7, Problem 4M, page 64]. Note that $\Sigma$ is completely normal and extremally disconnected. See also [7, Problem 6R, page 98] and [15, Example 3.1].

The point of this example is that $Y$ fails to be $T_{D}$. Recall that a space $X$ is $T_{D}$ if $(X \backslash \overline{\{x\}}) \cup\{x\}$ is open for each $x \in X$. The space $Y$ in the previous example is clearly $T_{0}$, but fails to be $T_{D}$ since $(Y \backslash \overline{\{\infty\}}) \cup\{\infty\}=\{\infty\}$ is not open in $Y$. However, if we restrict ourselves to $T_{D}$-spaces we have the following result:

Proposition 3.6. Let $X$ be a $T_{D}$-space. Then $X$ is perfect if and only if $O X$ is $F_{\sigma}$-perfect.

Proof. Let $U \in O X$. By hypothesis, there exists a countable family $\left(U_{n}\right)_{n \in \mathbb{N}}$ in $O X$ such that $\mathfrak{p}(U)=\bigvee_{n \in \mathbb{N}} \mathfrak{c}\left(U_{n}\right)$. Then $\mathfrak{c}\left(U_{n}\right) \subseteq \mathfrak{v}(U)$ for each $n \in \mathbb{N}$ and so it follows from property (P2) that $U \cup U_{n}=X$ for each $n \in \mathbb{N}$. Consequently, $\bigcup_{n \in \mathbb{N}}\left(X \backslash U_{n}\right) \subseteq U$.

On the other hand, let $x \in U$. Since $X$ is $T_{D}$ it follows that there exists an open $V \ni x$ such that $W=V \backslash\{x\}$ is open as well. We have that $U \rightarrow W \in \mathfrak{p}(U)=\bigvee_{n \in \mathbb{N}} \mathfrak{c}\left(U_{n}\right)$ and so there exists a countable family $\left(V_{n}\right)_{n \in \mathbb{N}}$ in $O X$ such that $U_{n} \subseteq V_{n}$ for each $n \in \mathbb{N}$ and

$$
U \rightarrow W=\bigwedge_{n \in \mathbb{N}} V_{n}=\operatorname{Int}\left(\bigcap_{n \in \mathbb{N}} V_{n}\right)
$$

Since $x \in U \cap V$ it follows that $U \cap V \nsubseteq W$ and thus $V \nsubseteq U \rightarrow W$, from which it follows that $x \notin U \rightarrow W$. Hence

$$
x \in X \backslash \operatorname{Int}\left(\bigcap_{n \in \mathbb{N}} V_{n}\right)=\overline{\bigcup_{n \in \mathbb{N}}\left(X \backslash V_{n}\right)} .
$$

Since $V$ is an open neighborhood of $x$ it follows that $V \cap\left(\bigcup_{n \in \mathbb{N}}\left(X \backslash V_{n}\right)\right) \neq \varnothing$. But $U \rightarrow W \subseteq \bigcap_{n \in \mathbb{N}} V_{n}$. Hence

$$
x \in \bigcup_{n \in \mathbb{N}}\left(X \backslash V_{n}\right) \subseteq \bigcup_{n \in \mathbb{N}}\left(X \backslash U_{n}\right) .
$$

In conclusion, $F_{\sigma}$-perfect locales model perfect spaces with the same proviso as in [15, III.7.2.1 (2) and III.7.3.1 (1)], that is, inside the class of $T_{D}$-spaces.

Recall that a locale $L$ is normal if $a \vee b=1$ implies that $a \vee u=1=b \vee v$ for some $u, v \in L$ satisfying $u \wedge v=0$. It follows from [9, Proposition 3.5] that the classes of 
$F_{\sigma}$-perfect locales and $G_{\delta}$-perfect locales coincide under normality. We include a direct proof here for the sake of completeness.

Proposition 3.7. A normal locale is $F_{\sigma}$-perfect if and only if it is $G_{\delta}$-perfect.

Proof. We only need to prove necessity. Let $L$ be a normal $F_{\sigma}$-perfect locale and $a \in L$. By hypothesis there exists a countable family $\left(a_{n}\right)_{n \in \mathbb{N}}$ in $L$ such that $\mathfrak{v}(a)=\bigvee_{n \in \mathbb{N}} \mathrm{c}\left(a_{n}\right)$. By (P2), $a \vee a_{n}=1$ for each $n \in \mathbb{N}$. Now, the normality of $L$ provides $u_{n}, v_{n} \in L$ such that

$$
a \vee u_{n}=1=a_{n} \vee v_{n} \quad \text { and } \quad u_{n} \wedge v_{n}=0, \quad n \in \mathbb{N} .
$$

It follows by (P2) that $\mathfrak{c}\left(a_{n}\right) \subseteq \mathfrak{v}\left(v_{n}\right)$. Moreover, $v_{n}<a$ and therefore, by (P3),

$$
\mathfrak{v}(a)=\bigvee_{n \in \mathbb{N}} \mathfrak{c}\left(a_{n}\right) \subseteq \bigvee_{n \in \mathbb{N}} \mathfrak{p}\left(v_{n}\right)=\mathfrak{p}\left(\bigvee_{n \in \mathbb{N}} v_{n}\right) \subseteq \mathfrak{v}(a) .
$$

Hence $a=\bigvee_{n \in \mathbb{N}} v_{n}$ with $v_{n}<a$ for each $n \in \mathbb{N}$. Finally, by (P2) and (P3),

$$
\mathfrak{c}(a) \subseteq \bigcap_{n \in \mathbb{N}} \mathfrak{p}\left(u_{n}\right) \subseteq \bigcap_{n \in \mathbb{N}} \mathfrak{c}\left(v_{n}\right)=\mathfrak{c}\left(\bigvee_{n \in \mathbb{N}} v_{n}\right)=\mathfrak{c}(a) .
$$

After all these considerations we drop the prefix $F_{\sigma}$ and introduce the following:

Definition 3.8. We call a locale perfect if each open sublocale is a join of countable many closed sublocales.

Then, we have the following (cf. [9, Propositions 3.5 and 4.2]):

Proposition 3.9. The following are equivalent for any locale $L$ :

(1) L is perfectly normal.

(2) L is a normal and perfect locale.

(3) For each $a \in L$ there is a countable family $\left(b_{n}\right)_{n \in \mathbb{N}}$ in $L$ such that $a=\bigvee_{n \in \mathbb{N}} b_{n}$ and $b_{n} \prec$ a for all $n \in \mathbb{N}$.

Remarks 3.10. (1) Perfect normality in pointfree topology was first considered by Charalambous [3] in the context of $\sigma$-frames. In [8], Gilmour observed that in the class of $\sigma$-frames perfect normality and regularity are equivalent concepts.

(2) Condition (3) was taken as the definition of a perfectly normal locale in [9]. In the terminology of [10], it says that every element in the locale is regular- $F_{\sigma}$ (i.e., a countable join of elements well inside it). Note that, for any topological space $X$, the regular- $F_{\sigma}$ elements of the locale $O X$ consist exactly of the regular- $F_{\sigma}$ subsets of $X$ (the complements of the usual regular- $G_{\delta}$ subsets of $\left.X[14]\right)$. It should be also noted that in the definition of a regular- $F_{\sigma}$ one may assume that each $b_{n}$ is regular. Indeed, $b_{n}<a$ implies $b_{n}{ }^{* *}<a$ and hence $a=\bigvee_{n \in \mathbb{N}} b_{n} \leq \bigvee_{n \in \mathbb{N}} b_{n}{ }^{* *} \leq a$. 
(3) For each regular- $F_{\sigma}$ element $a$, the closed sublocale $\mathfrak{c}(a)$ is a $G_{\delta}$-sublocale (and therefore the open sublocale $\mathfrak{v}(a)$ is an $F_{\sigma}$-sublocale). Indeed, if $a=\bigvee_{n \in \mathbb{N}} b_{n}$ with $b_{n}{ }^{*} \vee a=1$ for each $n \in \mathbb{N}$ then by (P2) and (P3) we get

$$
\mathfrak{c}(a) \subseteq \bigcap_{n \in \mathbb{N}} \mathfrak{v}\left(b_{n}{ }^{*}\right) \subseteq \bigcap_{n \in \mathbb{N}} \mathfrak{c}\left(b_{n}\right)=\mathfrak{c}\left(\bigvee_{n \in \mathbb{N}} b_{n}\right)=\mathfrak{c}(a)
$$

It is also easy to check that if we add normality to Proposition 3.6, then we can conclude (under $T_{0}$ ) that pointfree perfect normality, as normality, is a conservative extension of the classical notion, that is, a $T_{0}$ topological space $X$ is perfectly normal if and only if $O X$ is perfectly normal:

Proposition 3.11. Let $X$ be a topological space.

(1) If $X$ is perfectly normal, then $O X$ is perfectly normal.

(2) If $X$ is $T_{0}$, then $O X$ is perfectly normal if and only if $X$ is perfectly normal.

Proof. (1) follows from Proposition 3.4. Regarding (2), we first note that if $O X$ is perfect and normal, then it is subfit and thus, by [11, Lemma 2.4], it is a $T_{1}$ space (hence $T_{D}$ ). Finally, it follows from Proposition 3.6 that $X$ is perfectly normal.

\section{VARIANTS OF NORMALITY AND Oz LOCALES}

Now recall that a locale $L$ is almost normal (resp. mildly normal) if for any $a, b \in L$ satisfying $a \vee b=1$, with $a$ regular (resp. $a$ and $b$ regular), there exist $u, v \in L$ such that $u \wedge v=0$ and $a \vee u=b \vee v=1$ (note that it is redundant to impose here $u$ and $v$ to be regular since $u \wedge v=0$ iff $u^{* *} \wedge v^{* *}=0$ ).

We can now prove the following result which is directly related to Proposition 3.7:

Proposition 4.1. Let $L$ be a locale and let a be a regular element in $L$.

(1) If $L$ is almost normal, then $\mathrm{c}(a)$ is a $G_{\delta}$-sublocale if and only if it is an $F_{\sigma^{-}}$-sublocale.

(2) If $L$ is mildly normal, then $\mathfrak{c}(a)=\bigcap_{n \in \mathbb{N}} \mathrm{D}\left(a_{n}\right)$, with all $a_{n}$ regular, if and only if $\mathfrak{v}(a)=\bigvee_{n \in \mathbb{N}} \mathfrak{c}\left(a_{n}\right)$.

Proof. In both cases the proof of sufficiency follows the lines of that of Proposition 3.7 replacing normality by almost and mild normality, respectively.

By Proposition 3.9, a frame $L$ is perfectly normal if and only if any element in $L$ is regular- $F_{\sigma}$. We say now that a locale $L$ is perfectly mildly normal (or pm-normal for short) if any regular element in $L$ is regular- $F_{\sigma}$. Hence, pm-normal locales are to perfectly normal locales the same as mildly normal locales are to normal locales. Note that all the variants of normality we have considered are conservative extensions of their topological counterparts. 
Lemma 4.2. Suppose that $a, b \in L$ satisfy $a \vee b=1$ and that there exist two countable families $\left(a_{n}\right)_{n \in \mathbb{N}}$ and $\left(b_{n}\right)_{n \in \mathbb{N}}$ of regular elements such that

$$
\bigvee_{n \in \mathbb{N}} a_{n} \vee b=1=a \vee \bigvee_{n \in \mathbb{N}} b_{n},
$$

with $a_{n}<a$ and $b_{n}<b$ for every $n \in \mathbb{N}$. Then there exist $u, v \in L$ such that $u \wedge v=0$ and $a \vee u=1=b \vee v$.

Proof. Let

$$
u=\bigvee_{n \in \mathbb{N}}\left(b_{n} \wedge \bigwedge_{i=1}^{n} a_{i}^{*}\right) \quad \text { and } \quad v=\bigvee_{n \in \mathbb{N}}\left(a_{n} \wedge \bigwedge_{i=1}^{n} b_{i}^{*}\right)
$$

Then

$$
a \vee u=\bigvee_{n \in \mathbb{N}}\left(a \vee\left(b_{n} \wedge \bigwedge_{i=1}^{n} a_{i}^{*}\right)\right)=\bigvee_{n \in \mathbb{N}}\left(\left(a \vee b_{n}\right) \wedge\left(\bigwedge_{i=1}^{n}\left(a \vee a_{i}^{*}\right)\right)\right)=\bigvee_{n \in \mathbb{N}}\left(a \vee b_{n}\right)=1
$$

Similarly $b \vee v=1$. On the other hand,

$$
u \wedge v=\bigvee_{n \in \mathbb{N}} \bigvee_{m \in \mathbb{N}}\left(b_{n} \wedge \bigwedge_{i=1}^{n} a_{i}^{*} \wedge a_{m} \wedge \bigwedge_{i=1}^{m} b_{i}^{*}\right)=0
$$

since, for each pair of naturals $n, m$,

$$
b_{n} \wedge \bigwedge_{i=1}^{n} a_{i}^{*} \wedge a_{m} \wedge \bigwedge_{i=1}^{m} b_{i}^{*} \leq b_{n} \wedge \bigwedge_{i=1}^{m} b_{i}^{*} \leq b_{n} \wedge b_{n}{ }^{*}=0
$$

in case $n \leq m$ and

$$
b_{n} \wedge \bigwedge_{i=1}^{n} a_{i}^{*} \wedge a_{m} \wedge \bigwedge_{i=1}^{m} b_{i}^{*} \leq \bigwedge_{i=1}^{n} a_{i}^{*} \wedge a_{m} \leq a_{m}{ }^{*} \wedge a_{m}=0
$$

otherwise.

Lane proved in [13] that any pm-normal topological space is mildly normal. In our pointfree (and conservative!) setting we prove more with a much simpler proof.

Proposition 4.3. The following are equivalent for any locale $L$ :

(1) L is pm-normal.

(2) $L$ is mildly normal and for each regular element a in $L$ there exists a countable family $\left(a_{n}\right)_{n \in \mathbb{N}}$ of regular elements in L such that $\mathfrak{c}(a)=\bigcap_{n \in \mathbb{N}} \mathfrak{v}\left(a_{n}\right)$.

(3) $L$ is mildly normal and for each regular element $a$ in $L$ there exists a countable family $\left(a_{n}\right)_{n \in \mathbb{N}}$ of regular elements in $L$ such that $\mathfrak{v}(a)=\bigvee_{n \in \mathbb{N}} \mathfrak{c}\left(a_{n}\right)$.

Proof. (1) $\Longrightarrow$ (2): Let $a$ and $b$ be regular elements in $L$ such that $a \vee b=1$. By pmnormality, $a=\bigvee_{n \in \mathbb{N}} x_{n}$ and $b=\bigvee_{n \in \mathbb{N}} y_{n}$ with $x_{n}<a$ and $y_{n}<b$ for every $n \in \mathbb{N}$. Obviously the elements $a_{n}=x_{n}{ }^{*}$ and $b_{n}=y_{n}{ }^{*}$ satisfy the conditions of the Lemma 4.2 and thus there exist $u, v \in L$ such that $u \wedge v=0$ and $a \vee u=1=b \vee v$. Hence $L$ is 
mildly normal. On the other hand, for each regular element $a \in L$, by pm-normality, $a=\bigvee_{n \in \mathbb{N}} x_{n}$ with $x_{n}<a$ for every $n \in \mathbb{N}$. Hence, by (P2) and (P3),

$$
\mathfrak{c}(a) \subseteq \bigcap_{n \in \mathbb{N}} \mathfrak{p}\left(x_{n}{ }^{*}\right) \subseteq \bigcap_{n \in \mathbb{N}} \mathfrak{c}\left(x_{n}\right)=\mathfrak{c}\left(\bigvee_{n \in \mathbb{N}} x_{n}\right)=\mathfrak{c}(a) .
$$

(2) $(3)$ : This follows from Proposition 4.1 (2).

(3) $\Longrightarrow(1)$ : Let $a$ be a regular element in $L$. By hypothesis there exists a countable family $\left(a_{n}\right)_{n \in \mathbb{N}}$ of regular elements in $L$ such that $\mathfrak{D}(a)=\bigvee_{n \in \mathbb{N}} \mathrm{c}\left(a_{n}\right)$. Hence $a \vee a_{n}=1$ for each $n \in \mathbb{N}$. Since $L$ is mildly normal, it follows that there exist $u_{n}, v_{n} \in L$ such that $u_{n} \wedge v_{n}=0$ and $a \vee u_{n}=1=a_{n} \vee v_{n}$ for each $n \in \mathbb{N}$. Consequently (by (P2) and (P3) again),

$$
\mathfrak{D}(a)=\bigvee_{n \in \mathbb{N}} \mathfrak{c}\left(a_{n}\right) \subseteq \bigvee_{n \in \mathbb{N}} \mathfrak{p}\left(v_{n}\right) \subseteq \bigvee_{n \in \mathbb{N}} \mathfrak{c}\left(u_{n}\right) \subseteq \mathfrak{p}(a) .
$$

Locales where each regular element is a cozero element are called $O z$ locales and are the natural pointfree counterpart of Oz spaces. They were introduced in [2] and further studied in [1]. Recall that, by Proposition 2.3 of [1], a locale is $\mathrm{Oz}$ if and only if every element of the form $\bigvee_{n \in \mathbb{N}}\left(a_{n} \wedge b_{n}\right)$ with all $a_{n}$ and $b_{n}$ being regular is a countable union of elements well inside it.

The next result, which seems to have escaped to the authors of [1], shows that the class of $\mathrm{Oz}$ locales contains that of perfectly normal locales.

Proposition 4.4. A locale is $O z$ if and only if it is pm-normal.

Proof. Necessity is obvious. For sufficiency, let $a=\bigvee_{n \in \mathbb{N}}\left(a_{n} \wedge b_{n}\right)$ with all $a_{n}$ and $b_{n}$ being regular. By pm-normality, each regular element $L$ is regular- $F_{\sigma}$ and therefore $a_{n}=\bigvee\left\{x \in L^{*} \mid x<a_{n}\right\}$ and $b_{n}=\bigvee\left\{y \in L^{*} \mid y<b_{n}\right\}$ for each $n \in \mathbb{N}$. Then

$$
a=\bigvee_{n \in \mathbb{N}} \bigvee\left\{x \wedge y \mid x, y \in L^{*}, x<a_{n}, y<b_{n}\right\}
$$

For each such $x$ and $y$, we have that $x \wedge y \leq(x \wedge y)^{* *} \in L^{*}$ and $(x \wedge y)^{* *}<\left(a_{n} \wedge b_{n}\right) \leq a$ (since $\left.(x \wedge y)^{*} \vee\left(a_{n} \wedge b_{n}\right) \geq\left(x^{*} \vee a_{n}\right) \wedge\left(y^{*} \vee b_{n}\right)=1\right)$. Hence $a=\vee\left\{z \in L^{*} \mid z<a\right\}$.

Remarks 4.5. (1) Cozero elements are regular- $F_{\sigma}$, since $a \in \operatorname{Coz} L$ if and only if $a=$ $\bigvee_{n \in \mathbb{N}} a_{n}$ for some $a_{n} \ll a$ (where $\ll$ denotes the really inside relation [12]). The converse is obviously true in $\mathrm{Oz}$ locales.

(2) If $\prec$ is interpolative (e.g., if $L$ is a normal locale), then regular- $F_{\sigma}$ elements are cozero elements also. More generally, in any almost normal locale, each regular- $F_{\sigma}$ element belongs to $\operatorname{Coz} L$. In fact, for $a=\bigvee_{n \in \mathbb{N}} a_{n}$ with $a_{n} \prec a$ and $a_{n}$ regular, by almost normality there exist $u_{n}$ and $v_{n}$ such that $u_{n} \wedge v_{n}=0$ and $a_{n}^{*} \vee u_{n}=1=v_{n}^{*} \vee a$, hence $a_{n} \prec u_{n} \prec a\left(\right.$ since $\left.u_{n}^{*} \vee a \geq v_{n} \vee a=1\right)$. Then $a_{n} \ll a$. 


\section{IMAGES OF PERFECT LOCALES}

In this final section, we show that, as happens with normality, perfectness is an invariant property under closed maps, providing more evidence for our choice in Definition 3.8 .

We start by recalling from [15] that a localic map is a map $f: L \rightarrow M$ satisfying

(1) $f(\bigwedge S)=\bigwedge f(S)$ for any $S \subseteq L$,

(2) $f(a)=1$ implies that $a=1$, and

(3) $f\left(f^{*}(b) \rightarrow a\right)=b \rightarrow f(a)$ for every $a \in L$ and $b \in M$,

where $f^{*}$ denotes the left adjoint of $f$, that exists by condition (1). This left adjoint is a frame homomorphism (i.e., it preserves arbitrary joins and finite meets). A localic map $f$ is closed whenever the image of each closed sublocale of the domain is closed. In that case, $f[\mathfrak{c}(a)]=\mathfrak{c}(f(a))$.

Each localic map $f: L \rightarrow M$ induces the image map $f[-]: \mathcal{S}(L) \rightarrow \mathcal{S}(M)$, left adjoint to the preimage map $f^{-1}[-]: \mathcal{S}(M) \rightarrow \mathcal{S}(L)$.

Open sublocales are preserved by preimages. More specifically:

$$
f^{-1}[\mathfrak{p}(b)]=\mathfrak{o}\left(f^{*}(b)\right) \quad \text { for every } b \in M .
$$

Furthermore, if $f$ is surjective then the composite $f f^{-1}$ satisfies

$$
f f^{-1}[\mathfrak{o}(b)]=\mathfrak{o}(b) \quad \text { for every } b \in M .
$$

Indeed: the inclusion " $\subseteq$ " follows from the adjunction $f[-] \dashv f^{-1}[-]$; moreover, for each $b \rightarrow y$ in $\mathrm{v}(b)$, we have $b \rightarrow y=b \rightarrow f(a)=f\left(f^{*}(b) \rightarrow a\right.$ ) for some $a \in L$ (by ontoness of $f$ ) where $f^{*}(b) \rightarrow a \in \mathfrak{v}\left(f^{*}(b)\right)=f^{-1}[\mathfrak{p}(b)]$.

We can now prove that perfectnes is invariant under closed localic maps.

Proposition 5.1. Let $f: L \rightarrow M$ be a surjective localic map. If $f$ is closed and $L$ is perfect, then $M$ is also perfect.

Proof. Let $b \in M$. Since $L$ is perfect it follows that $f^{*}(b)=\bigvee_{n \in \mathbb{N}} c\left(a_{n}\right)$ for some countable family $\left(a_{n}\right)_{n \in \mathbb{N}}$ in $L$. Then, since $f[-]$ preserves arbitrary joins, we have

$$
\mathfrak{v}(b)=f f^{-1}[\mathfrak{v}(b)]=f\left[\mathfrak{v}\left(f^{*}(b)\right)\right]=f\left[\bigvee_{n \in \mathbb{N}} \mathfrak{c}\left(a_{n}\right)\right]=\bigvee_{n \in \mathbb{N}} f\left[\mathfrak{c}\left(a_{n}\right)\right]=\bigvee_{n \in \mathbb{N}} \mathfrak{c}\left(f\left(a_{n}\right)\right)
$$

Remark 5.2. Note that in general $f[-]$ does not preserve countable meets, so that the previous argument does not work if we replace perfectness by $G_{\delta}$-perfectness. This gives us one more argument for choosing $F_{\sigma}$-perfectness as the right way to extend the topological notion of perfectness to the pointfree setting.

Corollary 5.3. Let $f: L \rightarrow M$ be a surjective localic map. If $f$ is closed and $L$ is perfectly normal, then $M$ is also perfectly normal. 
Proof. Just combine the proposition above with the fact, proved in [10, Corollary 9.4], that normality is also invariant under closed localic maps.

\section{ACKNOWLEDGEMENTS}

The authors acknowledge financial support from the Ministry of Economy and Competitiveness of Spain (grants MTM2012-37894-C02-02 and MTM2015-63608-P), from the Basque Government (grant IT974-16) and from the Centre for Mathematics of the University of Coimbra (funded by the Portuguese Government through FCT/MEC and co-funded by the European Regional Development Fund through the Partnership Agreement PT2020). The paper was completed during a visit of JGG and JP to TK in Poznań. The hospitality of Adam Mickiewicz University is gratefully acknowledged.

\section{REFERENCES}

[1] B. Banaschewski, T. Dube, C. Gilmour and J. Walters-Wayland, Oz in pointfree topology, Quaestiones Math. 32 (2009) 215-227.

[2] B. Banaschewski and C. Gilmour, Oz revisited, In: Proceedings of the Conference Categorical Methods in Algebra and Topology, (H. Herrlich and H.-E. Porst, eds.), Math. Arbeitspapiere Nr. 54, pp. 19-23, Universität Bremen, 2000.

[3] M. G. Charalambous, Dimension theory for $\sigma$-frames, J. London. Math. Soc. (2) 8 (1974) 149-160.

[4] R. Engelking, General Topology, Heldermann Verlag, Berlin, 1989.

[5] T. Dube and O. Ighedo, Covering maximal ideals with minimal primes, Algebra Univers. 74 (2015) 411-424.

[6] T. Dube and O. Ighedo, More on locales in which every open sublocale is z-embedded, Topology Appl. 201 (2016) 110-123.

[7] L. Gillman and M. Jerison, Rings of continuous functions, Van Nostrand, 1960.

[8] C. R. A. Gilmour, Realcompact spaces and regular $\sigma$-frames, Math. Proc. Camb. Phil. Soc. 96 (1984) 73-79.

[9] J. Gutiérrez García, T. Kubiak and J. Picado, Pointfree forms of Dowker's and Michael's insertion theorems, J. Pure Appl. Algebra 213 (2009) 98-108.

[10] J. Gutiérrez García and J. Picado, On the parallel between normality and extremal disconnectedness, J. Pure Appl. Algebra 218 (2014) 784-803.

[11] J. Gutiérrez García, J. Picado and M. A. de Prada Vicente, Monotone normality and stratifiability from a pointfree point of view, Topology Appl. 168 (2014) 46-65.

[12] P. T. Johnstone, Stone Spaces, Cambridge Univ. Press, Cambridge, 1982.

[13] E. P. Lane, PM-normality and the insertion of a continuous function, Pacific J. Math. 82 (1979) 155162.

[14] J. Mack, Countable paracompactness and weak normality properties, Trans. Amer. Math. Soc. 148 (1970) 265-272.

[15] J. Picado and A. Pultr, Frames and locales: topology without points, Frontiers in Mathematics, Vol. 28, Springer, Basel, 2012. 
Department of Mathematics, University of the Basque Country UPV/EHU, Apdo. 644, 48080 Bilbao, SPAIN

E-mail address: javier.gutierrezgarcia@ehu.eus

Wydzią Matematyki i Informatyki, Uniwersytet im. Adama Mickiewicza, 61-614 Poznań, Poland

E-mail address: tkubiak@amu.edu.pl

CMUC, Department of Mathematics, University of Coimbra, 3001-501 Coimbra, Portugal

E-mail address: picado@mat.uc.pt 